\title{
ADAPTIVE SCHEMES FOR PACKET DATA IN A DS-CDMA ENVIRONMENT
}

\author{
Oriol Sallent, Ramon Agustí \\ Departament de Teoria del Senyal i Comunicacions \\ Universitat Politècnica de Catalunya \\ C/ Gran Capità s/n - 08034 Barcelona - Spain \\ Tel. +34-3-401.71.97 Fax +34-3-401.72.00 \\ E-mail : oriol @ xaloc.upc.es
}

\begin{abstract}
This paper presents a novel Adaptive DS-CDMA SlottedALOHA packet random access scheme for improving the throughput of the conventional DS-CDMA Slotted-ALOHA system. For this purpose a Mobile-assisted algorithm is envisaged to control the change of the transmission rate according to the traffic load. This algorithm revealed that the optimum behavior may be almost reached at a low complexity cost. Moreover, the proposed algorithm was found to be robust to intercell interference.
\end{abstract}

\section{INTRODUCTION}

Slotted ALOHA (S-ALOHA) has been widely recognized for packet radio applications when the data transmitted from the terminals are bursty or even recently for voice cellular communications [1]. S-ALOHA DS-CDMA scheme allows several users to transmit at the same time using a different spreading code for each one [2], [3], so that unsuccessful transmissions are caused entirely by multiple access interference, provided negligible thermal noise effects are assumed.

This paper is intended to improve the throughput achieved with a multi-receiver DS-CDMA S-ALOHA when a light load is offered to the system. For this purpose we will focus on the transmission rate used instead of the access protocol itself. In particular, different transmission rates are proposed to be used in order to utilize the most suitable rate according to the channel load at any time slot. Then, a fast and simple algorithm that command the processing gain of the Spread Spectrum DS scheme as a function of the channel load is envisaged. For low channel loads, a high CDMA processing gain is no longer necessary and it can be reduced so as to increase the actual bit rate. Therefore, in an extreme situation with a processing gain equal to one, all the users would access the same channel at their maximum bit rate according to a pure S-ALOHA.

In a real cellular scenario, interference from neighbough cells must be taken into account. To keep these interference at a reasonable level, the concept of cell cluster arises in techniques with a FDMA component. However, it is the case neither for DS-CDMA based systems nor for cellular S-
ALOHA [1], where a reuse factor of 1 is normally considered. Accepting that a throughput decrease will follow when considering the cellular structure, we are interested on assessing this decrease in terms of throughput as well as whether the proposed change of the transmission rate algorithm is still valid or not. We will show that no changes are required in the algorithm, despite the interference is not only dependent on the offered load but also on the propagation conditions (that strongly varies the interference level affecting to a reference user). Furthermore, some power control based policies are proposed to improve the system performance.

The paper is organized as follows. In Section 2 an analytical model for the DS-CDMA S-ALOHA system is presented, which is used to evaluate the optimum achievable throughput with an adaptive change of transmission rate algorithm. In Section 3 a particular S-ALOHA adaptive algorithm is proposed and assessed. In Section 4 a cellular model is considered and the impact of other-cell interference is attained. The change of the transmission rate algorithm is also studied under these conditions and limited-power strategies are evaluated. Conclusions close the paper in Section 5.

\section{MODEL FOR A S-ALOHA DS-CDMA ACCESS SYSTEM}

From now onwards $N$ registered users will be considered. These users can be in two different operation modes: "idle mode' and 'backlogged mode'. In the former mode there is no packet to be retransmitted and new packets are generated with probability $p_{o}$. Terminals enter the backlogged mode when an attempt to transmit a new packet fails. In this mode, the retransmission of the backlogged packet occurs in any given slot with probability $p_{r}$. While in the backlogged mode the user does not generate any new packet.

The process defined by the number of backlogged users at the beginning of the $k^{\text {th }}$ slot is a Markov chain, whose performance has been studied among others in [4]. When considering DS-CDMA, the formulation must be modified as it was done in [5]. Analytical throughput measurements can be obtained from the markovian model. 


\section{A. S-ALOHA DS-CDMA ACCESS SYSTEM}

A BPSK DS-CDMA access system with a processing gain given by $G_{p}$ is considered. All users have been assigned random PN signature sequences. A perfect power control capable of mitigating fadings of the channel is introduced. With a view to achieving this, a continuous link between BS and MS is needed so that the mobile transmits at rate $v$ when there is information to be sent and at rate $v^{\prime}$ (in general much lower) when the terminal is not active. $v^{\prime}$ should be high enough to allow the fading on the uplink path to be tracked by updating transmitted power in response to the BS commands.

An instantaneous power control permits a huge improvement in the system performance when compared with an open loop power control, and it is, in fact, considered in the already operative [6] and the proposed CDMA systems [7] respectively. Although an instantaneous power control could be envisaged for packet radio on a packet by packet basis [8], we have retained the continuous link approach because in this case no synchronization overhead at the beginning of time slot would be required, since the mobile is already synchronized. Whether continuous power control is retained or not it is in any case irrelevant for the proposed S-ALOHA scheme taken for granted that a throughput decrease should be considered in the non-continuous packet based power control approach.

\section{B. S-ALOHA DS-CDMA PERFORMANCE}

By assuming an ideal instantaneous power control, the channel can be seen as an AWGN if we use the gaussian hypothesis to model the interference originated by other users [9], [2]. Under these conditions the following expressions hold for the evaluation of the BER

$$
P_{b}(n)=Q\left(\sqrt{2 \frac{E_{b}}{N_{o}}}\right) ; \frac{E_{b}}{N_{o}}=\frac{1}{\frac{2(n-1)}{3 G_{p}}+\frac{2(N-n)}{3 G_{p}} \frac{v^{\prime}}{v}}
$$

where $n$ is the number of simultaneous users. The probability of detecting correctly a packet containing $\alpha B$ bits is

$$
P_{c}(n)=\left[1-P_{b}(n)\right]^{\alpha B}
$$

Keeping the transmission bandwidth constant, and taking as a reference a transmission rate of $v$ bits $/ s$, packet length of $\mathrm{B}$ bits and a processing gain $G_{p}$, the transmission rate could be increased by a factor $\alpha(\alpha>1)$ at the expense of a reduction in the spread capacity. That is, transmitting at rate $\alpha v$ bits $/ s$ would allow a processing gain of $G_{p} / \alpha$. However, in exchange for this reduction in processing gain, $\alpha B$ bits per time slot can be allocated instead of only $B$.

The achievable global throughput with different transmission rates obtained with the Markov model can be seen in Fig. 1, where $G$ stands for the offered load - average number of attempted transmissions per time slot -, and $p_{o}$ is set equal to $p_{r}$ so that $p_{0}=p_{r}=p=G / N, G$ is assumed to vary slowly. Moreover, for numerical results $v^{\prime}$ has been set to zero. Although there are no restrictions on the values of $\alpha$, for the sake of brevity and clarity only three rates $v, 2 v$ and $4 v$ bits $/ s$ $(\alpha=1,2,4)$ will be considered in the sequel.

It is clear from Fig. 1 that the higher rate $(4 v$ bits/s) is interesting when the system is lightly loaded: more bits per packet can be sent through the channel, since the little interference observed permits correct transmission. When the offered load increases so does the interference level, and therefore the rate $4 v \mathrm{bits} / \mathrm{s}$ is no longer interesting because of the higher BER compared to the $2 v$ bits $/ s$ rate. Using $2 v$ bits $/ s$ in this range is better than $4 v$ bits/s in the sense that a higher throughput is achieved although the packet contains half the number of bits per packet (errors occur quite often with $4 v$ bits $/ s$, but $2 v$ bits $/ s$ can still bear the interference because of the higher $G_{p}$ compared to that of $4 v \mathrm{bits} / \mathrm{s}$ rate). For higher offered loads the same trade-off appears between $2 v$ bits $/ s$ and ly bits/s.

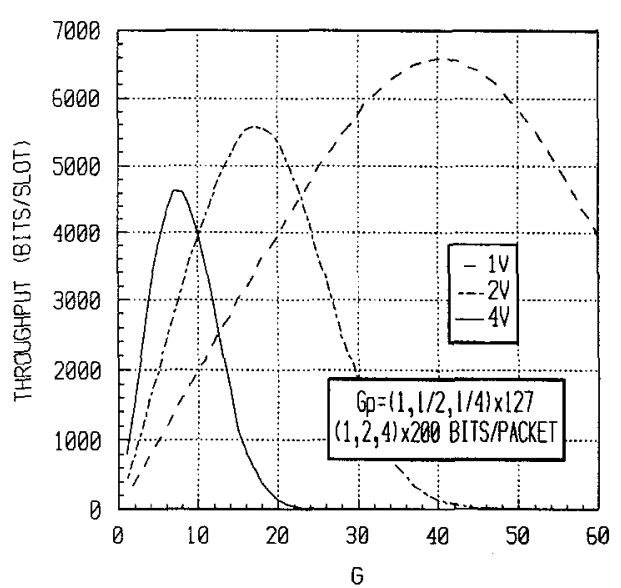

Fig. 1. Throughput performance for different transmission rates.

\section{ADAPTIVE S-ALOHA DS-CDMA}

In the light of the above results, an algorithm able to change the transmission rate used by the MS as a function of the traffic load of the system could be foreseen so that the maximum possible throughput could always be obtained.

The optimum throughput attained can be analytically obtained from the Markov model, and this result can be later used as a reference to assess the performance of the transmission rate change algorithm proposed below. In order to attain this optimum performance, the best combination of transmission rates should be obtained provided that $n$ simultaneous users are present. The resulting optimal table would be reached after an exhaustive search, as it is further explained in [10]. Fig. 2 shows this optimum throughput in comparison with the individual behaviors. 


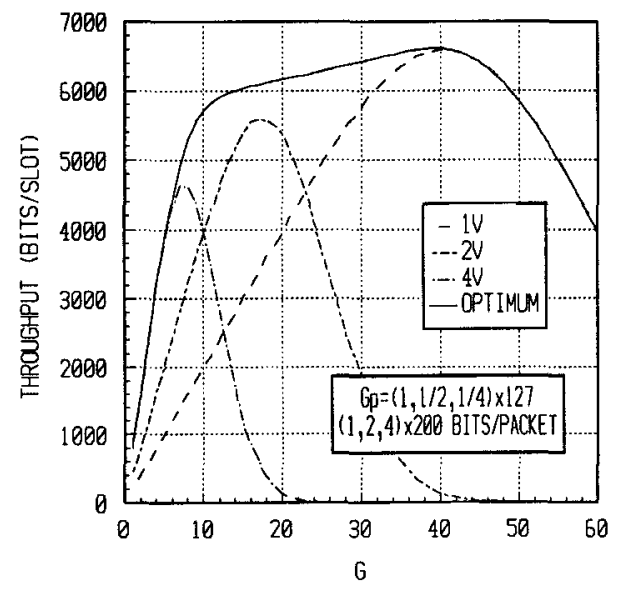

Fig. 2. S-ALOHA DS-CDMA optimum throughput.

\section{CHANGE OF THE TRANSMISSION RATE ALGORITHM}

In this section a proposal of an adaptive change of the transmission rate algorithm is addressed. The basic idea consist in sensing the traffic load through the channel in order to accommodate transmission rates accordingly.

The proposed simple algorithm carried out by the mobile station (MS) works as follows: each terminal traces its own evolution during the transmission time, that is, terminals count their successful and erroneous packets. In the absence of errors the mobile will assume a low traffic load and tries to use a higher transmission rate. The throughput should be increased in this way. If errors occur, the mobile decides that the channel is too loaded and tries a lower transmission rate. In this case fewer bits per packet are transmitted, but a global improvement of the throughput should also follow because these bits can be now detected correctly since processing gain increases accordingly.

Let us note that this decision is taken by the mobile without any exchange of information with the Base Station (BS) except for the packets acknowledgment. Even the MS does not need to indicate its choice of transmission rate before using it because the very same BS could be able to detect which one is arriving.

Specifically, the MS only needs to establish two parameters: the number of consecutive packet failures before changing to a lower rate (max_tr) and the number of consecutive packet successes before trying a higher rate ( $\min _{-}$suc).

Fig. 3 shows the throughput attained for $N=60$ registered mobiles. Fig. 1 has been taken as a reference in order to appreciate whether mobiles choose the most suitable rate or not. In spite of the simplicity of the algorithm, the envelope of the three individual graphs is almost reached, and this is not far from the optimum behavior. Results have been obtained with max_tr=1 and min_suc=7, which is the best choice, once other possibilities have been studied.

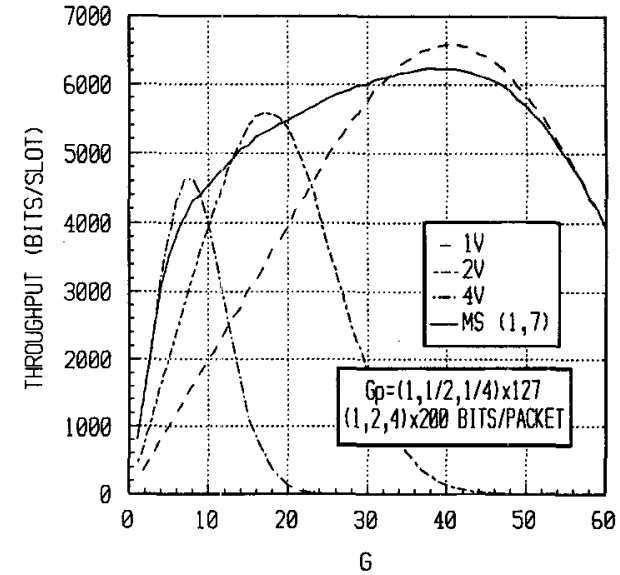

Fig. 3. Throughput performance for MS algorithm.

\section{MS ALGORITHM IN A CELLULAR ENVIRONMENT}

When considering multiple-cell CDMA systems, which will normally be the case, the situation becomes more complicated. There are basically two new problems that must be taken into account:

1-. The interference level originating from users in the other cells. This interference varies not only according to the attenuation in the path to the subscriber's cell site, but also inversely to the attenuation from the interfering user to his own cell site, which through power control by that cell site may increase or decrease the interference to the desired cell site.

2-. The necessary handover procedures. In order to keep the general interference level as low as possible, it would be desirable for each mobile to be attached to the cell site for which the radio path attenuation is minimized. Handover decisions are usually based on pilot-strength measurements (radio distance measurements). However, some time is necessary for power measurement averaging and for handover execution.

In order to evaluate the MS algorithm in a cellular environment several computer simulations have to be carried out, and the first requirement is to establish a channel model. The propagation attenuation is generally modeled as the product of a shadowing fading and a short-term fading. The former is due to the terrain configuration, whereas the latter is largely due to multipath reflections. An accepted representation for the received power is

$P_{r} \propto P_{t} \frac{\alpha^{2}}{r^{\mu} 10^{\varsigma / 10}}$

where $P_{r}$ is the received power, $P_{t}$ is the transmitted power, $\alpha^{2}$ is an exponential random variable, $\zeta$ is the $\mathrm{dB}$ attenuation due to shadowing, with zero mean and standard deviation $\sigma$ 
and the path loss decays with the $\mu$ th power of distance. The shadowing fading introduces slowly (or long-term) varying variations even for users in motion.

For simulation purposes a reference cell and its two-tier neighbour cells are also considered. The second-tier is only considered for reducing edge effects and for allowing mobiles in the first tier to be attached to such cell sites. Interference from second-tier to the reference cell is neglected, whereas the average value derived in [11] is assumed to affect first-tier cell sites. Different assumptions on the cellular model will be stated in the following subsections, where statistics are collected after 35,000 time slots.

\section{A. IDEAL ATTACHMENT}

As an upper bound on the throughput performance we consider the ideal situation where mobiles are always attached to the lowest attenuation cell site. Moreover, no Rayleigh fading is considered. Diversity as well as coding reduce the impact of the short-term fading, so it is common to omit the impact of Rayleigh fading [12]. Thus, the attenuation is affected only by a $\mu=4$ path loss law and a $6 \mathrm{~dB}$ shadow fading. $N=60$ subscribers for each reference cell and its firsttier cells are located randomly each time slot. Shadowing is also generated independently on a slot by slot basis. Mobile terminals are power limited, in such a way that at the edge of the cell site (which is normalized to unity) there is no margin for shadowing fading compensation. Therefore, the closed loop power control cannot be assured (in the sense that the received power from a subscriber under 'bad channel' may be below the desired level, $S)$. I $(r)$ being the interference level from a subscriber located at a distance $r$ from the reference cell, it holds that $I(r) / S \leq 1$.

Fig. 4 shows the performance attained. The throughput peaks for the individual transmission rates are around 30\% lower than the single cell case. The proposed algorithm performs the same as in the single cell case, attaining the envelope of the individual rate features.

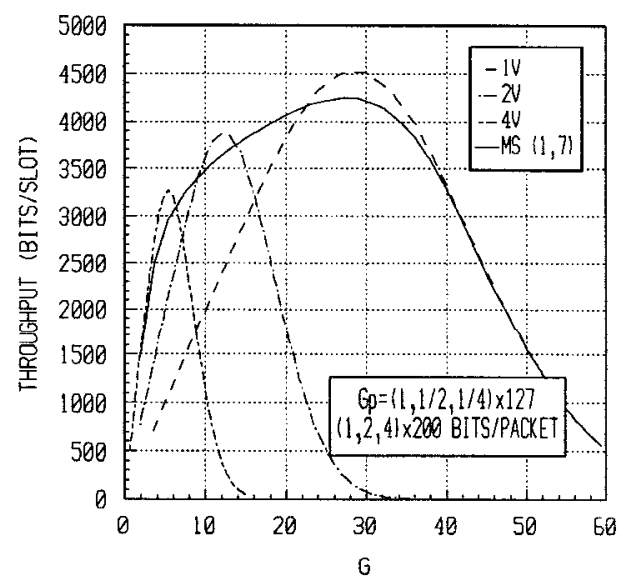

Fig. 4. MS algorithm in a cellular environment.

\section{B. NON-IDEAL ATTACHMENT}

The impact of short-term fading as well as the disadjustment arising from a handover execution will be covered in this subsection. Rayleigh is assumed to fade independently on a slot by slot basis, while log-normal shadowing is assumed to remain the same for 500 slots, which is coherent with [13] for typical values of the design parameters. As a representative result, we consider that 50 slots are required before a handover can be decided and executed when the long-term attenuation to the current cell site is not the lowest one of the surrounding cells. This time is necessary for averaging the Rayleigh fading and for channel allocation purposes. During this period the condition $I(r) / S \leq 1$ is not guaranteed. This model may in fact be too pessimistic, so it can be seen as a worst case on throughput performance.

It can be seen in Fig. 5 that the throughput peaks for the individual transmission rates are around 50-60\% lower than the single cell case. Performance of MS algorithm is also shown in Fig. 5, where the envelope of the individual transmission rates is again obtained. The only difference is that optimum parameters are $(2,7)$ instead of $(1,7)$. Setting max tr to 2 is better because it means that the mobile averages before taking a decision. So, it may avoid a rate reduction due to a single error caused by a strong random interference level.

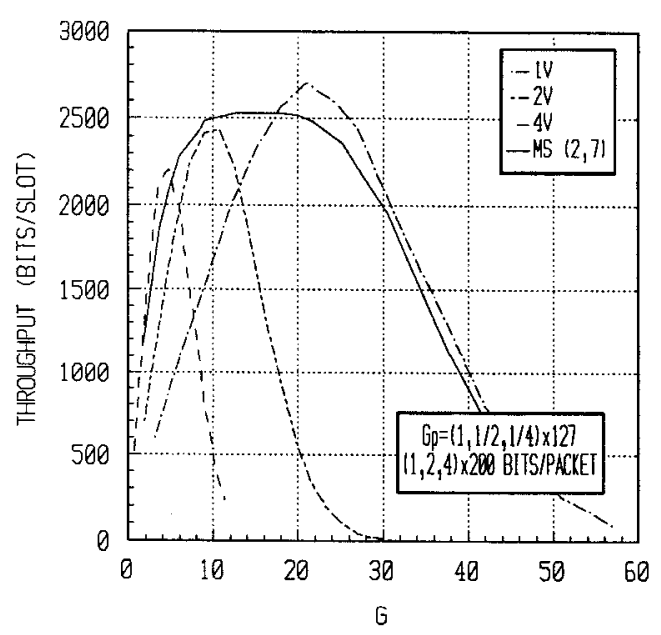

Fig. 5. MS algorithm in a cellular environment.

\section{POWER LIMITATION CONDITION}

Once the performance of a cellular DS-CDMA system has been assessed, a technique has been envisaged to improve it. Since the system is interference-limited, most of such techniques will try to reduce the interference level observed by a reference user. Besides some very well-known solutions like antenna sectoring or interference cancellation, it might be possible to exploit the role of the maximum transmitted power allowed (MAX_POW).

This parameter leads to a trade-off between the capacity to compensate fading at the base station receiver and the interference level. From a single user point of view, lowering 
MAX_POW means reducing the interference caused to both his own and the other cell sites. However, it makes it more difficult for the power control to maintain the signal reference level at the base station for the single user. The reverse applies if MAX_POW is increased. Which of these phenomena has more impact on the global throughput depends on the level to which the power is limited as well as on the offered load, which determines the total interference level.

Results for different values of MAX_POW are presented in Fig. 6. Only the performance of the MS algorithm is shown, and the gain obtained when limiting the maximum transmitted power can be noted. The throughput degradation for low loads when decreasing MAX_POW could be avoided by adaptively changing this parameter jointly with the transmission rate, according to the MS algorithm. Therefore, the interference effects of Rayleigh fading could in practice be strongly mitigated.

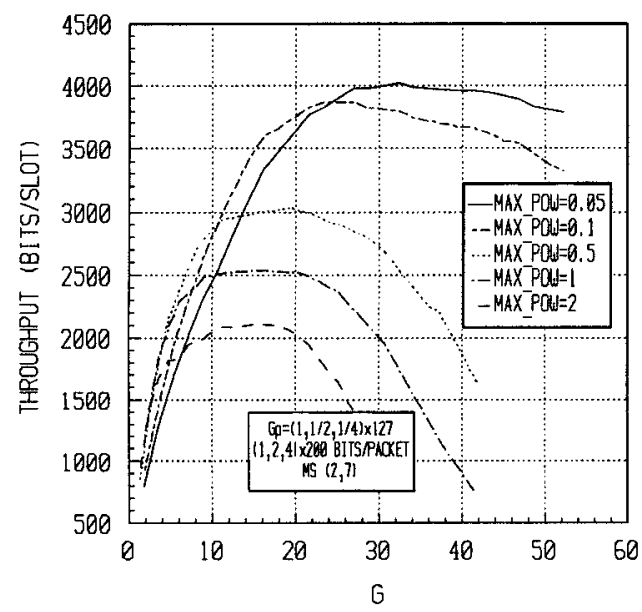

Fig. 6. A power limitation condition is imposed.

\section{CONCLUSIONS}

A new Adaptive DS-CDMA S-ALOHA technique for packet mobile communications access based on the choice of the most suitable transmission rate in any time slot has been addressed. The mobile assisted algorithm allows us to obtain almost the maximum attainable performance at a very low complexity cost, and by far outperforms those obtained with a conventional DS-CDMA S-ALOHA scheme. Simulations in a cellular environment show that the proposed algorithm is also robust to intercell interference. Moreover, limiting the maximum transmitted power proved to be a good policy for general throughput improvement.

\section{ACKNOWLEDGMENTS}

The work described in this paper was carried out within the CICYT TIC94-0870-C02-01 project, in the framework of National Plan of Spain.

\section{REFERENCES}

[1] M. ZORZI, S. PUPOLIN, "Slotted ALOHA for HighCapacity Cellular Communications", IEEE Transactions on Vehicular Technology, Vol. 43, No. 4, November 1994, pp. 1011-1021.

[2] D. RAYCHAUDHURI, "Performance Analysis of Random Access Packet-Switched Code Division Multiple Access Systems", IEEE Transactions on Communications, Vol. Com. 29, No. 6, June 1981, pp. 895-901.

[3] R. K. MORROW, J.S. LEHNERT "Packet Throughput in Slotted ALOHA DS/SSMA Radio Systems with Random Signature Sequences", IEEE Transactions on Communications, Vol. Com. 40, No. 4, July 1992, pp. 1223-30.

[4] L. KLEINROCK, S.S. LAM, "Packet Switching in a Multi-access Broadcast Channel: Performance Evaluation ", IEEE Transactions on Communications, Vol. Com.-23, No. 4, April 1975, pp.410-4.22.

[5] Z. LIU, M. EL ZARKI, "Performance Analysis of DSCDMA with Slotted ALOHA Random Access for Packet PCNs ", Wireless Networks 1 (1995), pp. 1-16.

[6] A. SALMASI, K. S. GILHOUSEN, "On the System Design Aspects of CDMA Applied to Digital Cellular and Personal Communications Networks", Proc. 41 $1^{\text {st }}$ IEEE Vehicular Technology Conference, St. Louis, May 1991.

[7] A. BAIER et al., "Design Study for a CDMA-Based Third-Generation Mobile Radio System ", IEEE Journal on Selected Areas in Communications, Vol. 12, No. 4, May 1994, pp. 733-743.

[8] M. J. McTIFFIN et al., "Mobile Access to an ATM Network Using a CDMIA Air Interface ", IEEE Journal on Selected Areas in Communications, Vol. 12, No. 5, June 1994, pp. 900-908

[9] M. B. PURSLEY, "Performance Evaluation for PhaseCoded Spread-Spectrurn Multiple-Access Communication Part I: System Analysis ", IEEE Transactions on Communications, Vol. Com.-25, No. 8, August 1977, pp.795-799.

[10] O. SALLENT, R. AGUSTI, "A Mobile Controlled Algorithm for Improving the Throughput in a S-ALOHA DSCDMA System", Proceedings of the 7th Personal, Indoor and Mobile Radio Communications (PIMRC'96), pp. 1192-96.

[11] M. KWOK, H. WANG, "Adjacent Cell Interference Analysis of Reverse-Link in CDMA Cellular Radio Systems" Vehicular Technology Conference VTC'95, pp.446-450.

[12] K. S. GILHOUSEN et al. , "On the Capacity of a Cellular CDMA System", IEEE Transactions on Vehicular Technology, Vol. 40, No. 2, May 1991, pp.303-312.

[13] M. GUDMUNDSON, "Correlation Model for Shadow Fading in Mobile Radio Systems", IEE Electronic Letters, Vol. 27, No. 23, 7th November 1991, pp. 2145-2146. 